\title{
Finally...
}

\section{Down the drain?}

There have been several reports of wound drains breaking during removal leaving part of the drain in situ. Surgeons should be aware that a drain may be weakened and liable to break if it is partially cut or nicked when sutured in position.

\section{It can happen!}

Despite rumour to the contrary, the electromagnetic interference from a mobile phone can, under certain circumstances, affect the performance of some devices. Definite reports of malfunction of infusion pumps have been reported to the Medical and Healthcare Products Regulatory Agency due to the proximity of a mobile phone. (The Programme Director at coress@btinternet.com would be interested to hear of any experience of the above.)

Reprinted from 'One Liners' (Issues $43 \& 44$, July \& September 2006) with the kind permission of the Medical and Healthcare Products Regulatory Agency.

\section{IMPORTANT OBSERVATIONS}

Surgeons are faced on a daily basis with living pathology and physiology. Accordingly, it is inevitable that from time to time, observations are made which lead to 'armchair hypothesising'. For example, many years ago, I was referred two women who had suffered seat belt injury to their breasts (one was the driver and the left breast was involved, the other a passenger and the right breast was involved). The trauma was severe and, within 6 months, each developed cancer in the traumatised breast. Could trauma be a cause of breast cancer I hypothesised? What a ridiculous suggestion you might say - but what if...?

Most hypotheses based on anecdotal observations are nonsense but, just occasionally, a gem develops, e.g. Fleming and penicillin. It is also possible that surgeons are too embarrassed to mention such observations and certainly do not have the facilities or expertise to develop and investigate their pet hypothesis.

I invite surgeons who have made an 'outrageous' observation resulting in a 'ridiculous' hypothesis to submit their 'concept' to the Annals. It will be subjected to expert peer review and, should it be deemed of possible importance or worthy of confirmation, will be published in the Annals. Such articles should be no more than 200 words or 150 words if accompanied by a figure. Don't be shy your observation may have clinical importance and could generate a hypothesis worthy of confirmation. 